\title{
LA COMPRENSIÓN Y LA INFERENCIA EN EL ESTUDIO DE HIPERTEXTOS CON EL APOYO DE UN AGENTE GENERADOR DE PREGUNTAS
}

\author{
Luis Facundo Maldonado G., Luis Carlos Sarmiento V., \\ Luis Bayardo Sanabria R., Nerey Ortega del Castillo y David Macías M. ${ }^{11}$
}

RESUMEN

Este documento es una síntesis del proyecto "Agentes de software generadores de preguntas y el desarrollo de la competencia cognitiva de hacer inferencias: la comprensión y la inferencia en diferentes niveles de profundidad en ambientes hipermediales", desarrollado por el Grupo TECNICE de la Universidad Pedagógica Nacional de Colombia.

La investigación analiza el efecto del uso de un agente de software generador de preguntas durante la etapa de estudio de un hipertexto en el desarrollo de competencias cognitivas de hacer inferencias a diferentes niveles, por parte de estudiantes de educación media.

Hacer preguntas es la función de un agente artificial y se evalúa su efecto en el proceso de razonamiento de un agente natural. La generación de preguntas sirve al agente natural para que identifique lo que necesita aprender (Ram, 1991). En esta lógica se desarrolla un agente artificial que formula preguntas sobre el dominio de conocimiento de la geografía considerando tres niveles jerárquicos de la ontología usada en la representación.

Se compara el efecto producido en el desarrollo de la competencia cognitiva de hacer inferencias de sujetos que estudian un hipertexto diseñado con base en una estructura ontológica y otros que estudian el mismo hipertexto con el generador de preguntas que opera en cuatro configuraciones: generación de preguntas de primero, segundo o tercer nivel y configurable a voluntad del usuario.

El estudio muestra un efecto muy significativo de la ontología utilizada en la representación de conocimiento y del modelo adaptativo, sobre la capacidad de hacer inferencias, en contraste con la formulación de preguntas controlada por el computador. Contrario a lo esperado, el entrenamiento en preguntas de alto nivel no genera diferencias significativas con respecto al entrenamiento con preguntas de más bajo nivel y los resultados dan pie para sostener la hipótesis de efecto específico por nivel de entrenamiento.

Palabras clave: competencia cognitiva, inferencias, ontología, ambientes hipermediales.

11 Investigadores del Grupo teCnICE, Profesores Universidad Pedagógica Nacional de Bogotá. 


\begin{abstract}
This document summarizes the results of a research project, named "Question generator software agents and the cognitive skill of making inferences: comprehension and inference at different levels, in hypermedia environments", developed by the TECNICE Group at the Universidad Pedagogica Nacional in Bogotá, Colombia.
\end{abstract}

We analyze the effect of using a question generator agent, during the study stage, on cognitive skill development of making inferences at three levels, by middle school students.

An artificial software agent plays the role of making questions and its effect on the natural agent reasoning process is assessed. Question production helps the natural agent to identify what to learn (Ram, 1991). Following this approach, the software agent is able of generating questions on the geography knowledge domain based on three levels of the ontology used in the representation.

The effect on the cognitive skill development of making inferences of students which studied a hypertext based on an ontological structure is compared with that of students which studied the same hypertext using a question generator agent in three arrangements: first, second an third level of questions, and user controlled system.

This study shows a strong effect of the ontology used in the knowledge representation and the adaptive model, in the development of inference skill, compared with the questions controlled by the computer. Contrary to our expectations, students which tried high level questions did not achieve better than those who tried lower level questions and the data support the hypothesis of a specific effect of each question level on the inference learning.

Key words: cognitive skill, inferences, ontology, hypermedia environments

Recibido: 3 de abril de 2009

Aceptado: 23 de octubre de 2009

\title{
INTRODUCCIÓN
}

La comprensión de la dinámica del aprendizaje usando hipertextos constituye el elemento principal de motivación para desarrollar este proyecto. La estructura implícita en un hipertexto funciona como una base de interacción de un agente artificial que reconoce e interpreta las estructuras ontológicas que existen en el hipertexto. Un agente artificial actúa en el hipertexto buscando estructuras de bajo nivel para ejecutar operaciones estructurales representadas en preguntas. Un hipertexto basado en una ontología muestra contenidos con diferentes niveles de agregación, de tal manera que los lectores se formen modelos mentales, dependiendo de la comprensión del contenido del hipertexto (Marshall \& Frank,1993). El hipertexto se considera como un ambiente de aprendizaje que ayuda a los estudiantes a ver las interrelaciones conceptuales importantes mejorando el aprendizaje de conocimiento 
complejo (Jonassen \& Wang, 1993). En esta dimensión, el trabajo intenta mostrar el proceso de razonamiento de un usuario a partir de la taxonomía del hipertexto (Millard, Moreau, Davis \& Reich, 2000). La tarea consiste en generar preguntas de diferentes niveles de inferencia que conduzcan al lector a un proceso de navegación a través de conjuntos de nodos traslapados en la estructura antológica jerárquica. Las categorías ontológicas y el agente generador de preguntas constituyen dos elementos valiosos hipotéticamente para potenciar el aprendizaje autónomo en escenarios hipertextuales.

La dimensión conceptual del documento trata tres aspectos principales: la concepción ontológica en el diseño hipertextual, el rol de la pregunta en el proceso de aprendizaje y el modelamiento de agentes generadores de preguntas. La parte investigativa analiza el comportamiento de estas estructuras en la comprensión y producción de inferencias.

\section{Ontologías y agentes artificiales}

Nuestro acercamiento sigue la lógica formulada por Minsky (1974). Hay indicios que muestran consistentemente que el ser humano procesa bloques estructurados de información, en razón de que la percepción y la memoria de trabajo tienen una capacidad de procesamiento limitada, lo que obliga a organizar bloques o estructuras. Se ha demostrado que la memoria de trabajo tiene un límite cercano a siete unidades funcionales y que cuando se supera este número su capacidad de recuerdo disminuye drásticamente; ésta, por otra parte, es independiente del tamaño o cantidad de información que agrupan estas unidades (Miller, 1956). Una estrategia efectiva, por tanto, para incrementar la capacidad de procesamiento de información, consiste en agrupar la información en unidades funcionales (Murdock, 1963 y 1969).

Bien puede pensarse que estas unidades se tratan como sistemas y que su tamaño depende del número de relaciones y elementos que intervienen y que, a su vez, los elementos de estas estructuras se piensan como sistemas analizables de la misma manera. En una estructura se anidan, entonces, otras estructuras. La diferenciación de éstas es equivalente a su nivel de análisis (figura 1). La atención del agente procesador de información puede centrarse en la estructura general como conjunto o en cualquiera de los nodos.

La economía de pensamiento consiste en procesar estructuras de diferentes niveles de integración: aquellas que tienen como elementos estructuras entre las cuales establecen relaciones y cada uno de estos elementos, a su vez, puede ser percibida y procesada como una estructura de más bajo nivel. A este tipo de jerarquías las llamamos ontologías. Un agente inteligente representa de esta manera el mundo que conoce para desarrollar consecuentemente su comportamiento adaptativo.

El supuesto subyacente a nuestro trabajo en relación con la comprensión de la autonomía en el aprendizaje, se puede expresar así: si un estudiante desarrolla una ontología para representar un dominio de conocimiento y tiene una representación de la ontología misma —nivel metacognitivo - la capacidad de regulación de sus procesos de búsqueda se puede mejorar. La ontología es una forma de organización de conocimiento que procede por niveles 
de agregación o integración. Un nivel más alto incluye más subsistemas, un nivel más bajo incluye más especificaciones. El nivel de mayor integración es también un nivel de mayor generalidad y el más bajo es de mayor especificidad.

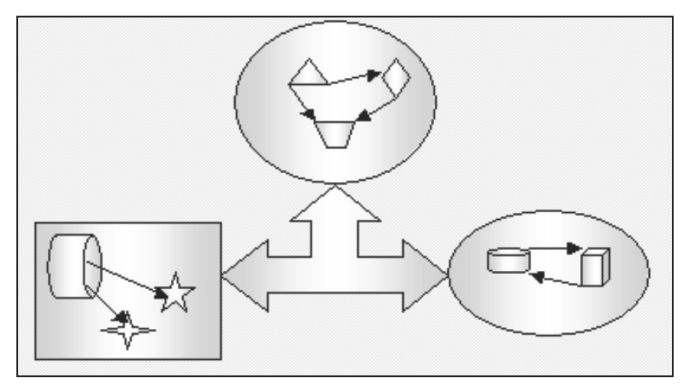

Figura1. Un sistema visto como conjunto de estructuras anidadas. Los componentes en cada nodo se integran en un todo mediante relaciones. Los niveles de investigación son vistos como niveles de agregación que favilitan el procedimiento de información. (Tomado de "Ambiente para el Diseño del Aprendizaje Significativo" Maldonado et al., 2001)

En Maldonado, et. al., (2001), se desarrolla un sistema que bien se puede clasificar en la categoría de Instrumento Cognitivo. Este programa — SIMAS - permite generar sistemas de marcos en la lógica planteada por Minsky (1974). Paralelamente se construye el prototipo de un programa generador de preguntas que usa como fuente un hipertexto y que cumple también las características de ser instrumento con el cual aprender.

Los sistemas de marcos comparten las propiedades fundamentales con los sistemas de redes semánticas y constituyen también una forma de ontología. El Generador de Preguntas no sirve como instrumento para construir una ontología, sino que utiliza una ontología para construir sus preguntas.

\section{AGENTE GENERADOR DE PREGUNTAS -AGP-}

\section{Arquitectura de un Agente}

Un agente está organizado jerárquicamente por procesos cognitivos como percepción, razonamiento, acción y registro en memoria. Los procesos de percepción adquieren, abstraen y filtran datos sensoriales antes de ser enviados a otros subprocesos. Los sistemas de acción controlan la ejecución de acciones externas en los efectores.

La percepción puede influenciar sobre la acción directamente a través de arcos reflejos o a través de procesos de coordinación de acciones percibidas. Los sistemas cognitivos interpretan percepciones, solucionan problemas, hacen planes y guían estrategias perceptivas y acciones externas juntas. Estos procesos operan concurrente y asincrónicamente y se comunican pasando mensajes. Las operaciones de percepción y acción ocurren en menor tiempo que las operaciones cognitivas. 


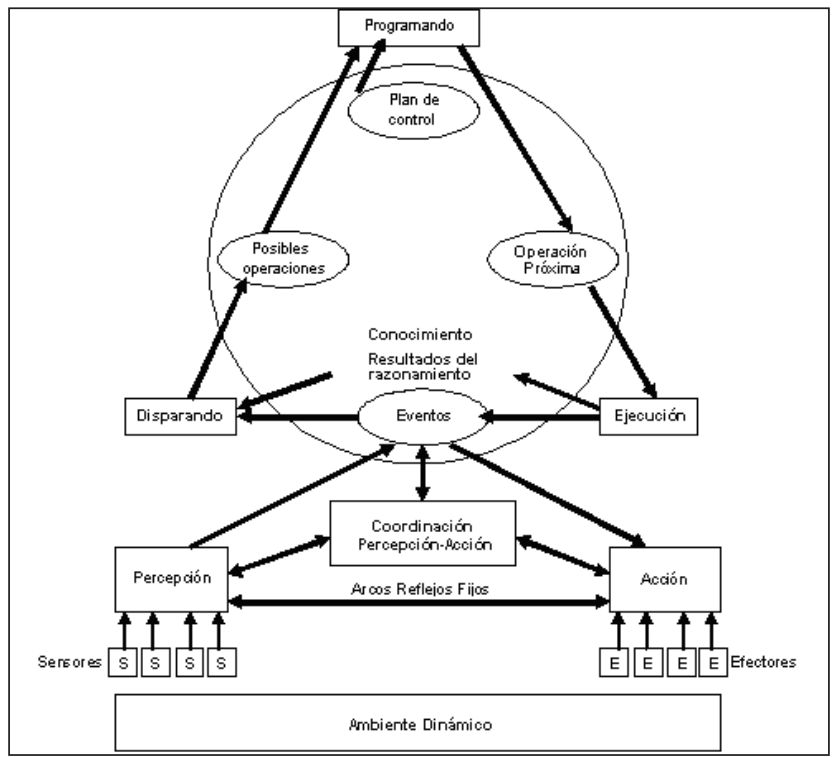

Ilustración 1. Arquitectura de un agente (Adaptado de: A Blackboard Architecture for Control, Artificial Intelligence. Hayes-Roth.1985)

El sistema cognitivo, en lo más sustancial, se puede diseñar siguiendo una forma de tablero extendido para soportar planes dinámicos de control. En este caso, la arquitectura del agente tiene las siguientes características (Erman, Hayes-Roth, Lesser \& Reddy, 1980):

- Las entradas perceptuales y las operaciones internas de razonamiento producen cambios globales en la memoria.

- Cada evento semejante desencadena un número de operaciones posibles de razonamiento.

- Las operaciones posibles están programadas para ejecutarse con base en planes de control activo.

- Los planes de control son construidos, así mismo, y modificados por operaciones de razonamiento.

- Las acciones posibles y los planes de control son representados en un lenguaje interpretable por la máquina que soporta semánticas parciales del mismo juego de acciones para realizar estos planes.

Un agente de software es un sistema computarizado que está situado en algún ambiente o programa de computador y que es capaz de realizar alguna acción en forma flexible y autónoma en este ambiente para lograr los objetivos para los cuales fue diseñado (Wooldridge, 1997). Un agente se alimenta de conceptos aplicados usualmente por humanos y tiene el poder de cambiar en alguna forma su ambiente (Pfeifer, 2001). Para Franklin \& Graesser (1996), un agente autónomo hace parte del ambiente y actúa sobre éste. Los agentes de software normalmente sirven como asistentes a los humanos en tareas específicas. Idealmente, un agente que funcione continuamente, sería capaz de aprender de su experiencia. 


\section{El rol de la pregunta en el aprendizaje}

Estudios realizados sobre el rol de la pregunta en el aprendizaje sugieren que ésta cumple tres funciones: establece y mantiene la atención, facilita la codificación y permite la conjetura (Wager \& Wager, 1985). La generación de preguntas es el proceso de identificación de lo que un agente natural necesita aprender (Ram, 1991). El aprendizaje se incrementa a medida que las preguntas son respondidas a través de la experiencia de interacción de los sujetos con el ambiente de aprendizaje. Las preguntas surgen de la interacción entre los intereses y objetivos de un agente natural y la información provista por el ambiente de aprendizaje. Las preguntas persiguen explicaciones que son estructuras de conocimiento que permiten a un sujeto responder sus cuestionamientos basados en el entendimiento causal de una situación. La comprensión de un agente natural se puede explicar como el proceso de relatar lo que éste lee, para las preguntas que el agente artificial genera. Estas preguntas representan el objetivo de conocimiento del agente natural; ejemplo, las cosas que éste desea aprender (Ram, 1991).

Tabla 1. Funciones de la pregunta en el aprendizaje

\begin{tabular}{|c|c|}
\hline PROCESO DE APRENDIZAJE & ROL DE LA PREGUNTA \\
\hline \multirow{2}{*}{$\begin{array}{l}\text { Recepción de estímulos por } \\
\text { parte del aprendiz }\end{array}$} & Capta la atención \\
\hline & Crea incertidumbre \\
\hline \multirow[t]{2}{*}{$\begin{array}{l}\text { Registro de información a } \\
\text { través de los órganos sensoriales }\end{array}$} & $\begin{array}{l}\text { Crea expectativa para la tarea de } \\
\text { aprendizaje }\end{array}$ \\
\hline & $\begin{array}{l}\text { Activa procesos de control en } \\
\text { la ejecución (West, Farmer \& } \\
\text { Wolff, 1991) }\end{array}$ \\
\hline \multirow[t]{3}{*}{$\begin{array}{l}\text { Percepción selectiva para } \\
\text { almacenamiento en MCP }\end{array}$} & $\begin{array}{l}\text { Actúa como prerrequisito para } \\
\text { recordar en la memoria de } \\
\text { corto plazo }\end{array}$ \\
\hline & Escanea \\
\hline & Atención selectiva \\
\hline \multirow{3}{*}{$\begin{array}{l}\text { Ensayo para mantener } \\
\text { información en la MCP }\end{array}$} & Ensaya (Kumar, 1971) \\
\hline & $\begin{array}{l}\text { Mantiene y renueva los ítems } \\
\text { almacenados en MCP (Miller,1956) }\end{array}$ \\
\hline & Sintetiza y encadena \\
\hline \multirow{3}{*}{$\begin{array}{l}\text { Codificación semántica para } \\
\text { almacenamiento en MLP }\end{array}$} & Codificación \\
\hline & Acreención (Ausubel,1968) \\
\hline & $\begin{array}{l}\text { Preguntas que producen aprendi- } \\
\text { zajes integrando nuevo conoci- } \\
\text { miento dentro de las estructuras } \\
\text { existentes o creando nuevas }\end{array}$ \\
\hline
\end{tabular}




\begin{tabular}{|l|l|}
\hline \multirow{2}{*}{$\begin{array}{l}\text { Recuperación de la MLP a } \\
\text { la memoria de trabajo }\end{array}$} & Recupera, generaliza en la práctica \\
\cline { 2 - 2 } & $\begin{array}{l}\text { Activa la organización de la } \\
\text { respuesta }\end{array}$ \\
\cline { 2 - 2 } & Prueba para comprender o no \\
\hline $\begin{array}{l}\text { Generación de respuestas } \\
\text { a los efectores }\end{array}$ & $\begin{array}{l}\text { Provee indicaciones para futuras } \\
\text { representaciones }\end{array}$ \\
\cline { 2 - 2 } & Provee refuerzos \\
\cline { 2 - 2 } & Provee información correctiva \\
\hline Representación en el \\
ambiente del aprendiz & Sirve de pos test o test de retención \\
\cline { 2 - 2 } & $\begin{array}{l}\text { Es una oportunidad para reforzar } \\
\text { y/o corregir debido a la variación } \\
\text { en contexto }\end{array}$ \\
\hline $\begin{array}{l}\text { Control de procesos a } \\
\text { través de estrategias ejecutadas }\end{array}$ & Afina esquemas existentes \\
\hline
\end{tabular}

El aprendizaje es un proceso de búsqueda de respuestas a preguntas que representan lo que un agente natural necesita para realizar una tarea. Las preguntas constituyen los objetivos específicos de conocimiento del agente. Según Ram (1991), el aprendizaje es un proceso activo que involucra la generación de preguntas y la búsqueda de inferencias en el contexto. En un modelo de procesamiento de información, el aprendizaje ocurre en un número de estados secuenciales (Bangert-Drowns et al.; Kumar, 1971; Gagné, 1985; Atkinson \& Shiffrin, 1968). En este enfoque, la investigación típicamente indaga sobre los efectos de la pregunta en uno o varios estados.

\section{Activación de conocimiento previo y cambios conceptuales}

Biemans y Simons (1999) consideran que el uso del conocimiento previo puede ser una estrategia clave para construir representaciones mentales ricas y útiles y que, en consecuencia, un ambiente de aprendizaje basado en computador, que active el conocimiento previo del estudiante, podría ayudar a reestructurar este conocimiento — cambio conceptual- y construir uno nuevo con mejor comprensión de contexto. En consecuencia, desarrollan un sistema llamado CONTACT que toma como dominio la Geografía Física. La estrategia del sistema, muestra fuerte capacidad para direccionar el procesamiento de información. Los investigadores concluyen que un programa de computador que ayuda al estudiante a identificar y contrastar sus preconceptos influye positivamente en la compresión de los textos. Un instrumento útil es el de la presentación de preguntas de escogencia múltiple combinada con la presentación de explicaciones y ejemplos y que le ayuda a razonar sobre los criterios que hacen que una respuesta pueda ser correcta o errónea.

Maldonado, Ortega, Sanabria, Macías, González, (2001), (2001: c) desarrollaron una versión de agente generador de preguntas con la siguiente estructura de diálogo: 
- Soy tu amigo Preguntón. Contrastemos tu lectura y mi lectura.

- Hagamos preguntas. Si contestamos todas las preguntas correctamente hacemos moñona y nuestro puntaje será igual al número de respuestas al cuadrado. De otra forma, el puntaje será igual al número de respuestas correctas.

- Cuántas preguntas de 2 a 7 crees contestar sin fallar

- «Pregunta»

- Hagamos conjeturas: Pensemos una posible respuesta.

- Califica como verdadera o falsa la respuesta. «Respuesta hipotética»

- Ahora contrastemos tu respuesta con la mía

- Yo pienso que es «Respuesta»

- Ahora vamos a la página donde encontramos la información «Página»

Este trabajo muestra que quienes responden correctamente preguntas, obtienen mejores resultados en pruebas de comprensión, unos días después de estudiar un texto. También es importante anotar que en esta investigación se utilizan dos formas de agente preguntón: uno activo y otro pasivo. Con esta configuración del ambiente se observa que quienes tuvieron el preguntón pasivo también activaron al generador de preguntas y alcanzaron un nivel que estadísticamente es equivalente al de quienes usaron la primera forma de preguntón. Esto evidencia en los estudiantes que responden preguntas una actividad muy atractiva y de gran utilidad en su aprendizaje.

\section{Aprendizaje de inferencias}

Una ontología de representación sirve de base para la ordenación. A partir de la ontología se establecen diferentes tipos de inferencias. Por ejemplo, se pueden tener dos proposiciones que establezcan relaciones causales y con base en ellas determinar la valoración de proposiciones intermedias. Las organizaciones sistémicas o espaciales pueden también servir de base para activar la competencia cognitiva de inferir.

Maldonado, et. al., (2003) estudian el desarrollo de competencias de hacer inferencias en el contexto de sistemas de clasificaciones —ontología jerárquica—por acción de un agente de software activador de procesos de lectura. El puntaje en el juego de hacer ordenamientos es predictor del rendimiento en una prueba sobre inferencias. Estos resultados hacen pensar que el estudio de información organizada siguiendo una ontología jerárquica incide en el aprendizaje de competencias cognitivas de hacer inferencias.

Frank, et. al. (2003) desarrollan un modelo matemático para el estudio de inferencias desde la perspectiva literaria de la narrativa. Típicamente se proponen dos proposiciones y se pide que se generen proposiciones intermedias para que las dos primeras sean coherentes. Ejemplo, "1. El director y el camarógrafo se estaban preparando para la filmación de la actriz en el borde del techo del piso catorce del edificio de la historia cuando súbitamente se cayó la actriz. 2. Sus hijas huérfanas acusaron al director y al personal del estudio por negligencia". Posibilidades de proposiciones intermedias serían: 1. La caída de la actriz le causó la muerte. 2. La actriz sufrió un paro cardíaco y murió. 3. La actriz cayó del $14^{\circ}$ piso al primer piso. 
El proceso de inferencia valora las proposiciones intermedias. La inferencia lógica puede considerar en las dimensiones sintáctica o formal y semántica o de significado. La lógica se preocupa por la organización formal y por el conjunto de reglas que hacen que el valor de las proposiciones inferidas a partir de un conjunto de premisas, sea válido. Cuando la lógica se aplica en la práctica entra en juego el valor semántico.

\section{DESCRIPCIÓN DEL SOFTWARE}

\section{EI hipertexto y la ontología}

El software consta de dos partes principales. Un hipertexto sobre el tema Producto Interno Bruto (PIB) y un Agente Generador de Preguntas (AGP) - Figura 2.

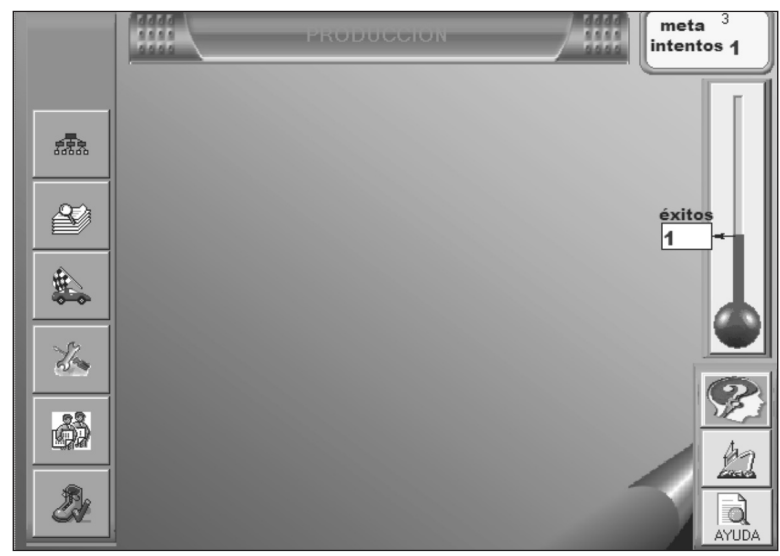

Figura 2. Pantalla principal. Arriba el nombre del nodo, la meta como número de preguntas que el estudiante quiere responder y el número actual de intentos. A la izquierda los botones que activan la información de las ranuras.A la derecha el termómetro que muestra el número de éxitos, el botón Preguntón que activa el generador de preguntas, el botón de acceso al mapa o Navegador y la Ayuda en línea.

El tema del PIB se estudia normalmente en noveno grado de educación básica en el sistema educativo colombiano. El hipertexto está programado siguiendo una ontología jerárquica que se proyecta sobre un sistema de marcos. La tabla 2 muestra las siete categorías de ranuras con base en las cuales se describe cada nodo.

Las categorías "Ejemplo" y "Es una subcategoría de” son vínculos dinámicos entre nodos. A través de ellas se puede navegar por el hipertexto. Su dirección es inversa, de tal manera que si A tiene como "Ejemplo" a A1, entonces por este vínculo se viaja de A a A1 y, en consecuencia, A1 "Es una subcategoría" de A y por este vínculo se puede regresar de A1 a A. 
Tabla 2. Sistema de ranuras en el hipertexto

\begin{tabular}{|l|l|l|}
\hline \multicolumn{1}{|c|}{ NOMBRE DE RANURA } & & \\
\cline { 1 - 1 } $\begin{array}{l}\text { Ejemplo } \\
\text { Es una subcategoría de }\end{array}$ & & \\
\cline { 1 - 1 } Quienes realizan & & \multirow{2}{*}{ Explicación de la ranura } \\
\cline { 1 - 1 } Finalidad & & \\
\cline { 1 - 1 } Cómo se hace & & \\
\hline Lo que se obtiene & & \\
\hline La cantidad de & & \\
\hline
\end{tabular}

Las otras ranuras son descriptores que dan información sin llevar a otro nodo. El nombre de la ranura suministra una información de orden categorial y abstracto. El contenido de la ranura, en contraste, provee información específica, vinculando el nombre del nodo — por ejemplo, "Extracción de recursos naturales" - con el nombre de la ranura- por ejemplo, "Finalidad"La explicación de la ranura es una información equivalente al contenido, pero, en un formato más extenso (Tabla 2).

\section{AGP: Modelo de procesamiento de preguntas}

El AGP es un agente generador de preguntas que utiliza un sistema de matriz para representar los nodos con sus diferentes ranuras. Inicialmente esta matriz contiene 0 en todas sus celdas. Para generar una pregunta, el AGP selecciona el nodo con el criterio del valor más bajo o el valor 0 en la celda que lo representa y, de manera similar, una ranura en el nodo seleccionado. Cuando el usuario da una respuesta correcta, la celda correspondiente incrementa su valor en 1. Con esta estrategia el AGP propone al usuario, estudiar la información menos conocida. Partiendo del nodo seleccionado procede a configurar el problema de acuerdo al nivel de inferencia.

El AGP presenta expresiones como la siguiente:

\section{'Quiénes realizan' producción cultivos transitorios es: agricultores e industria agrícola.}

En la expresión “Quienes realizan" es nombre de ranura; "Producción de cultivos transitorios" es nombre de nodo; "agricultores e industria agrícola" es el contenido de la ranura. Esta estructura es una forma típica orientada para que el estudiante adquiera familiaridad con las estructuras de marcos. El uso de colores se hace para resaltar las categorías de la representación.

Las preguntas se categorizan en tres niveles (Figura 3):

1. Preguntas de nivel 1 de inferencia. Para este nivel, el AGP procede a formular una proposición tomando información del nombre del nodo y de la ranura seleccionada. Luego, puede aplicar un cuantificador, un operador lógico y pide al usuario que califique como 
verdadera o falsa la proposición. Para decidir tiene la opción de revisar la información en el nodo correspondiente.

2. Preguntas de nivel 2 de inferencia. En este nivel, el AGP evalúa una información teniendo en cuenta la categoría superior de la cual el nodo es subcategoría. La proposición se genera a partir de la información del nodo seleccionado y la otra a partir del nodo padre o categoría inmediatamente superior.

3. Preguntas de nivel 3 de inferencia. En el tercer nivel, el AGP selecciona un nodo de bajo puntaje en éxitos del usuario y evalúa si tiene un abuelo o categoría con dos niveles de superioridad. Luego genera una proposición para cada uno de estos nodos y pide al usuario que las califique como verdaderas o falsas.

El siguiente es un ejemplo de la forma como el AGP genera las preguntas:

Ejemplo 1: Todo continente tiene escala muy baja en extracción de recursos naturales.

Antecedente: La finalidad en producción cultivos permanentes es: cultivando plantas con periodos de vida superiores a un año.

Posible inferencia: 'La finalidad' en producción cultivos permanentes es: cultivando plantas con periodos de vida superiores a un año, o la explicación de cómo 'se hace' producción cultivos permanentes es: preparando el terreno, sembrando, desyerbando y cuidado de las plantas para evitar las plagas y enfermedades en su largo ciclo de vida.

El AGP toma un nombre de ranura (La finalidad), una preposición (en) un nombre de nodo (producción de cultivos permanentes), el verbo ser, el contenido de la ranura (cultivando plantas con período de vida superiores a un año). De esta manera construye una proposición y pide al usuario que la evalúe como verdadera o falsa. En la segunda parte, el AGP construye dos proposiciones vinculadas con el conectivo lógico "o" y pide evaluar si de la primera parte se puede inferir la segunda. En este caso el usuario necesita conocer tanto el valor de verdad de las proposiciones como las condiciones formales de una proposición compleja o molecular.

\section{Población, muestra y procedimientos experimentales}

El ambiente de aprendizaje se probó con 130 estudiantes de noveno grado de educación básica. El contenido de este programa es parte del currículo regular en geografía de este grado. Los estudiantes se distribuyeron aleatoriamente en cinco grupos. El grupo E que tiene acceso sólo al hipertexto estuvo en una sala diferente a los otros grupos. Las sesiones de estudio fueron simultáneas para todos los grupos y en el mismo colegio. 


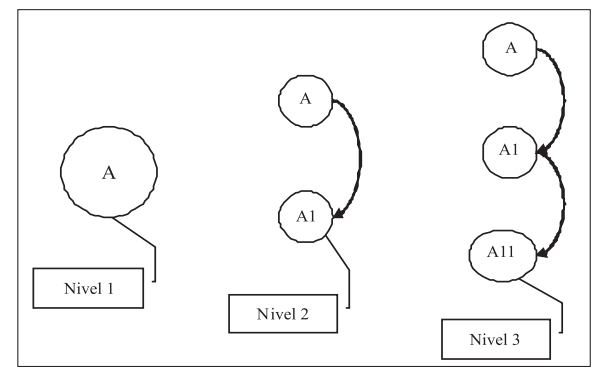

Figura 3. Niveles de inferencia. Nivel 1: Valor de la proposición en el nodo A. Nivel 2: valor de una proposición en A1 relacionando con A, de la cual es subcategoría. Nivel 3: Valor de la proposición en A11, relacionando con A1 de la cual es subcategoría y con A, de la cual A1 es subcategoría.

Con los estudiantes se desarrollaron las siguientes sesiones:

1. Primera sesión de estudio del software según las condiciones del grupo asignado

2. Presentación de evaluación al terminar la sesión

3. Presentación de una prueba de retención a los cuatro días

4. Sesión de estudio, una vez presentada la prueba de retención

5. Presentación de prueba de evaluación cinco minutos después de terminar la segunda sesión de estudio

El sistema registró el tiempo de las sesiones de estudio, el total de preguntas, el total de respuestas a preguntas en sesión de estudio, el total de respuestas correctas en la evaluación sumativa después de la sesión de estudio y el total de respuestas por nivel en cada una de las evaluaciones.

Gráfica 1. Comparación de medias mediante el análisis de varianza usando como variable dependiente el total de éxitos en el cuestionario de evaluación y como covariables el total de preguntas respondidas en sesión de estudio, el total de respuestas correctas y el tiempo de estudio $\mathrm{A}=$ con preguntas de $1 \mathrm{er}$. nivel; $\mathrm{B}=$ con preguntas de $2^{\circ}$ nivel; $\mathrm{C}=$ con preguntas 3 er nivel; $\mathrm{D}=$ configuración.

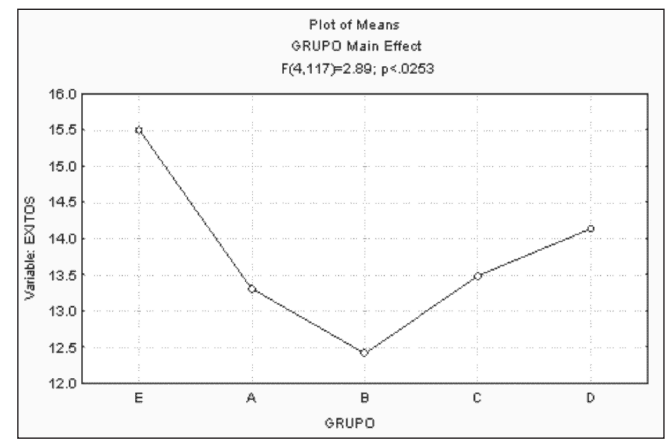




\section{Discusión de resultados}

\section{Relación entre nivel de inferencias activadas por las preguntas del AGP y la competencia de hacer inferencias de diferente nivel}

Al diseñar esta investigación, nuestro razonamiento partía de la consideración de que la economía de pensamiento consiste en procesar estructuras que establezcan relaciones a diferentes niveles de integración de la información.

En consecuencia, esperábamos que un agente, que hiciera preguntas a un nivel alto, indujera al estudiante a construir estructuras de conocimiento de mayor complejidad. Así, los estudiantes que utilizaran un agente de software que hiciera preguntas de nivel alto obtendrían mejores resultados en un test que midiera competencias cognitivas de comprensión e inferencia que quienes utilizaran un agente que generara preguntas de nivel más bajo. Es decir, que el Grupo C puntuaría más alto que el $\mathrm{B}$, y éste que el C.

Gráfica 2. Comparación del rendimiento global en la prueba final, después de la segunda sesión de estudio.

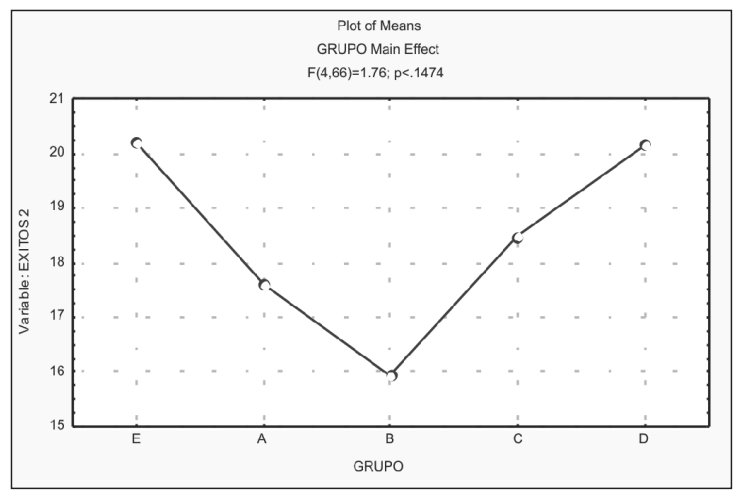

Si tomamos como base los datos globales en la evaluación al final de la primera sesión de estudio (Gráfica 1), encontramos que $\mathrm{C}$ tiende a ser superior a los grupos A y B; B no es superior al A; pero, estas diferencias no son estadísticamente significativas, de tal manera que esta suposición no se ve plenamente confirmada desde esta perspectiva. Exactamente la misma relación se da en la prueba de retención y en la prueba final después de la segunda sesión de estudio (Gráfica 2).

Si se toman los resultados referidos a las inferencias de segundo nivel, en la primera evaluación el grupo B que estudió con preguntas de segundo nivel únicamente, tiene puntajes inferiores a los grupos A y C, pero en la prueba de retención supera al grupo A y reduce las diferencias con el grupo $\mathrm{C}$ y después de la segunda sesión de estudio aumenta la ventaja frente al grupo A y reduce aún más las diferencias con el grupo C. Este fenómeno sería argumento 
para sustentar que el Grupo B madura más lentamente la curva de aprendizaje y que al final estaría mostrando mejores resultados que los otros grupos en inferencias de segundo nivel.

Tabla 3. Matriz de correlación de las variables dependientes en las diferentes mediciones del experimento. Correlación significativa a nivel de $\mathrm{p}<0.01$. Número de casos $=93$

\begin{tabular}{|c|c|c|c|c|c|c|c|c|c|c|c|c|c|}
\hline \multirow[b]{2}{*}{$\begin{array}{l}\infty \\
\frac{1}{2} \\
\frac{1}{0} \\
\frac{1}{5} \\
\frac{1}{\infty}\end{array}$} & \multirow{2}{*}{$\begin{array}{c}\text { SESIONES } \\
\text { PARÁMETROS }\end{array}$} & \multicolumn{8}{|c|}{ 1era. sesión } & \multicolumn{4}{|c|}{ 2da. sesión } \\
\hline & & 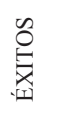 & $\sum^{\vec{y}}$ & 勇 & 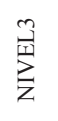 & 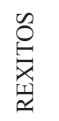 & 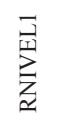 & 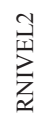 & 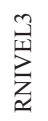 & 足 & 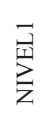 & 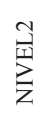 & $\stackrel{3}{M}^{3}$ \\
\hline \multirow{8}{*}{ 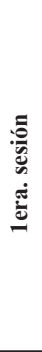 } & ÉXITOS & 1.00 & .75 & .71 & .80 & .50 & .32 & .37 & .42 & .49 & .31 & .33 & .50 \\
\hline & NIVEL 1 & .75 & 1.00 & .35 & .44 & .45 & .36 & .36 & .30 & .36 & .26 & .31 & .31 \\
\hline & NIVEL 2 & .71 & .35 & 1.00 & .29 & .39 & .24 & .37 & .27 & .36 & .27 & .21 & .37 \\
\hline & NIVEL 3 & .80 & .44 & .29 & 1.00 & .34 & .19 & .17 & .38 & .41 & .21 & .25 & .46 \\
\hline & REXITOS & .50 & .45 & .39 & .34 & 1.00 & .76 & .75 & .80 & .56 & .41 & .43 & .52 \\
\hline & RNIVEL 1 & .32 & .36 & .24 & .19 & .76 & 1.00 & .46 & .43 & .42 & .39 & .25 & .38 \\
\hline & RNIVEL 2 & .37 & .36 & .37 & .17 & .75 & .46 & 1.00 & .40 & .35 & .28 & .32 & .27 \\
\hline & RNIVEL 3 & .42 & .30 & .27 & .38 & .80 & .43 & .40 & 1.00 & .58 & .35 & .43 & .59 \\
\hline \multirow{4}{*}{ 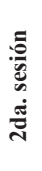 } & ÉXITOS & .49 & .36 & .36 & .41 & .56 & .42 & .35 & .58 & 1.00 & .75 & .75 & .89 \\
\hline & NIVEL 1 & .31 & .26 & .27 & .21 & .41 & .39 & .28 & .35 & .75 & 1.00 & .38 & .51 \\
\hline & NIVEL 2 & .33 & .31 & .21 & .25 & .43 & .25 & .32 & .43 & .75 & .38 & 1.00 & .50 \\
\hline & NIVEL 3 & .50 & .31 & .37 & .46 & .52 & .38 & .27 & .59 & .89 & .51 & .50 & 1.00 \\
\hline
\end{tabular}

Si se consideran los datos referidos a inferencias de tercer nivel, el grupo tres muestra medias consistentemente superiores a los otros grupos. Lo cual sería argumento a favor de la especificidad de nivel de las preguntas de entrenamiento sobre el aprendizaje de inferencias. La matriz de correlaciones que se muestra en la Tabla 4 daría un argumento a favor de la especificidad del nivel, por cuanto el rendimiento en la primera prueba, en el Nivel 3 y Nivel 2 no son predictores del rendimiento en los niveles inferiores en la prueba de retención. Pero, podría explicarse en razón de que la curva de aprendizaje aún está en evolución y las relaciones cambian al establecer relaciones entre la prueba de retención y la prueba final.

La tesis de la especificidad del entrenamiento por nivel se puede sustentar mostrando que durante todo el proceso evaluado los resultados del Grupo D, que dispuso del AGP adaptativo y que pudo estudiar preguntas de los tres niveles, fueron superiores - en algunos casos superando el nivel de significación- a los de los grupos A, B y C, con entrenamiento en preguntas de un solo nivel.

Si bien hay argumentos para sustentar la tesis de la especificidad del nivel de entrenamiento, en contra de la tesis de que entrenar en pruebas de alto nivel es suficiente para que el estudiante desarrolle inferencias de niveles más bajos, las diferencias encontradas entre las medias no son estadísticamente significativas, y se requiere mayor investigación para sustentar esta tesis. 


\section{Contraste entre sólo hipertexto y AGP adaptativo}

Se partió en el diseño del supuesto de que la pregunta, como un activador del conocimiento previo, es una estrategia clave para construir representaciones mentales ricas y significativas. En consecuencia, se esperaba que un ambiente de estudio con un agente generador de preguntas potenciara la generación de conocimiento estructurado y el desarrollo de competencias de comprensión e inferencia.

En las condiciones de la investigación se esperaba que el Grupo E que estudió con el hipertexto sin preguntas tuviera una media inferior al grupo D que tuvo un AGP adaptativo, es decir, en el cual el usuario pudo programar la proporción de preguntas de cada nivel para su estudio.

Los datos globales de las evaluaciones al final de la primera prueba, muestran alguna superioridad del Grupo E (Tabla 4), sin que sea una diferencia estadísticamente significativa, en prueba de retención y en prueba final después de la segunda evaluación; las medias son estadísticamente iguales para los dos grupos (Tabla 4).

Tabla 4. Resumen de medias según grupos y mediciones para cada variable dependiente. M1 = primera evaluación; Rtn = segunda evaluación o de retención; M2 = Evaluación después de la segunda sesión de estudio.

\begin{tabular}{|c|c|c|c|c|c|c|c|c|c|c|c|c|c|}
\hline & \multicolumn{3}{|c|}{$\begin{array}{c}\text { INFERENCIAS } \\
\text { NIVEL 1 }\end{array}$} & \multicolumn{3}{c|}{$\begin{array}{c}\text { INFERENCIAS } \\
\text { NIVEL 2 }\end{array}$} & \multicolumn{3}{c|}{$\begin{array}{c}\text { INFERENCIAS } \\
\text { NIVEL 3 }\end{array}$} & \multicolumn{5}{c|}{ TOTAL } \\
\hline Gr & M1 & Rtn & M2 & M1 & Rtn & M2 & M1 & Rtn & M2 & M1 & Rtn & M2 & N \\
\hline E & 4.7 & 6.0 & 6.3 & 5.1 & 5.4 & 5.6 & 5.2 & 7.1 & 9.1 & 15.0 & 18.7 & 21.1 & 19 \\
\hline A & 4.6 & 5.6 & 6.0 & 4.2 & $\mathbf{4 . 9}$ & $\mathbf{4 . 7}$ & 4.3 & 4.9 & 6.4 & 13.2 & 15.3 & 17.1 & 20 \\
\hline B & 4.3 & $\mathbf{5 . 3}$ & $\mathbf{5 . 2}$ & 3.8 & 5.2 & 5.9 & 4.3 & 5.5 & 6.1 & 12.5 & 16.0 & 17.2 & 18 \\
\hline C & 5.0 & $\mathbf{6 . 0}$ & $\mathbf{5 . 8}$ & 4.5 & 5.7 & 6.1 & 4.5 & 5.7 & 6.7 & 14.0 & 17.8 & 18.6 & 20 \\
\hline D & 5.2 & $\mathbf{6 . 7}$ & $\mathbf{6 . 1}$ & 5.1 & 6.0 & 7.1 & 4.3 & 6.1 & 7.2 & 14.6 & 18.8 & 20.4 & 16 \\
\hline
\end{tabular}

Los resultados no permiten sustentar una superioridad del sistema con sólo hipertexto frente al sistema con AGP adaptativo, ni la relación inversa. El hecho de que sea en la tercera medición cuando se encuentren diferencias significativas, hace pensar un efecto de entrenamiento en uso del AGP.

El hipertexto fue diseñado con base en una ontología jerárquica probada en una investigación anterior (Maldonado, et. al, 2001). Este tipo de estructura facilita la comprensión de la información por parte del usuario. Por otra parte, el manejo de la estructura de la pregunta como es administrada por el AGP requiere esfuerzo inicial y una mayor inversión de tiempo, factores que inciden en que sea más rápida la lectura del hipertexto sin AGP y que los resultados de éste se vean más tardíamente. 


\section{Preguntas respondidas correctamente y aprendizaje}

Las investigaciones anteriores dan pie para afirmar que las preguntas resueltas satisfactoriamente tienen un impacto fuerte en la orientación del procesamiento de información. En consecuencia se esperaría que haya una correlación significativa y positiva entre el número de preguntas respondidas correctamente en sesión de estudio y puntajes obtenidos en un test de rendimiento.

Los datos por niveles muestran correlación significativa y de signo negativo en inferencias de nivel 1 al final de la primera y segunda sesiones de estudio, muy cercana al nivel de significación en retención de inferencias de nivel 3. La correlación es también significativa con los resultados en inferencias de nivel 3 al final de la segunda sesión de estudio.

La correlación de respuestas correctas en la primera sesión de estudio es significativa y de signo positivo con respecto a los resultados globales en la prueba general al final de la primera y de la segunda sesión de estudio y notoria o cercana al nivel de significación con respecto a la prueba de retención.

Los datos muestran que las preguntas y las respuestas correctas de la segunda sesión de estudio no tienen relación significativa con el rendimiento en las pruebas en contraste con las preguntas y respuestas en la primera sesión de estudio. Esto hace pensar que su efecto se pierde en la medida en que se avanza en la curva de aprendizaje o que hubo algún factor que debilitó su efecto.

Estos resultados replican conclusiones obtenidas en investigaciones anteriores que muestran que las preguntas respondidas correctamente en sesión de estudio se relacionan positivamente con los resultados en evaluaciones, pero, al mismo tiempo sugieren estudiar la evolución de su efecto dentro de la dinámica de maduración de la curva de aprendizaje.

\section{CONCLUSIONES}

\section{Maduración de la curva de aprendizaje}

Los resultados muestran un incremento entre la prueba aplicada inmediatamente después de la primera sesión de entrenamiento y la prueba aplicada dos días después. Thiede, Anderson \& Therriault (2003) señalan que los investigadores controlan las condiciones de estudio y no permiten que los estudiantes desarrollen su proceso y que las pausas entre el entrenamiento pueden favorecer el recuerdo.

Koriat \& Shitzer-Reichert (2002) encuentran que la introducción de pausas, entre las sesiones de entrenamiento y la evaluación, mejora el recuerdo, aunque disminuyen el nivel de precisión. Los resultados de esta investigación muestran que los estudiantes consistentemente mejoran su nivel de recuerdo frente a la evaluación hecha inmediatamente después de la sesión de estudio. 
Gráfica 3. Análisis de varianza de medidas repetidas. Evolución de la curva de aprendizaje a través de tres mediciones. Nivel_1=prueba al finalizar la primera sesión de estudio; Nivel_2= Prueba de retención; Nivel_3= Prueba al finalizar la tercera sesión de estudio. Var.1= resultados en la prueba general.

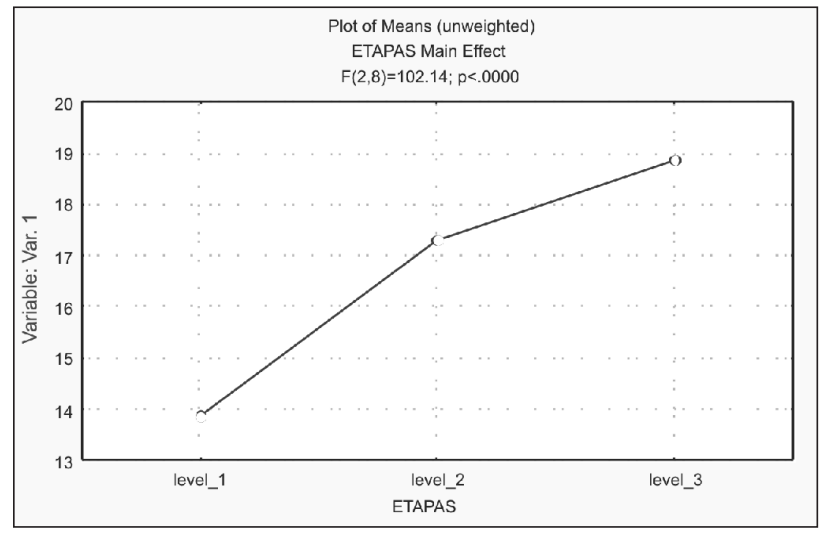

Por otra parte, el incremento del aprendizaje durante la segunda sesión de estudio muestra que con excepción del aprendizaje de las habilidades para hacer inferencias de primer nivel, las demás habilidades no han llegado a su nivel máximo de maduración con la primera sesión de estudio, y cabe pensar que, en algunos casos, se requeriría entrenamiento adicional (Gráfica 3).

El modelo que se introdujo en esta investigación concuerda con estudios previos sobre juegos de descubrimiento donde se observa un fenómeno similar Maldonado, et.al., (1999). La investigación sobre ambientes de aprendizaje basados en computador, pero también la práctica educativa en general, basa sus juicios sobre los métodos de enseñanza sobre condiciones en las cuales los estudiantes no han logrado estabilizar la evolución de su aprendizaje.

Esto nos lleva a pensar que la única variable dependiente a tener en cuenta no es el aprendizaje en un tiempo fijo, sino tomar criterios de maduración de la curva de aprendizaje en relación con el esfuerzo y el tiempo invertidos por el estudiante y evaluar cuál es el nivel de estabilización de la curva en cada ambiente. Estos criterios permiten evaluar a profundidad la calidad de los ambientes de aprendizaje.

\section{Efecto de las preguntas y las respuestas correctas en sesión de entrenamiento sobre el aprendizaje}

Los resultados de esta investigación concuerdan con Maldonado, et.al., (2003), donde se encuentra que las respuestas correctas a los problemas de comprensión (primer nivel de inferencias) se correlacionan positivamente con los resultados en pruebas de evaluación. El aporte de esta investigación en particular está en mostrar que también se da un efecto en los diferentes niveles de inferencia y que el efecto de la primera sesión de estudio se observa aún después de la segunda del mismo. 
Esta investigación no controló la dimensión formal o estructural de la pregunta frente a la dimensión semántica $\mathrm{y}$, posiblemente, los estudiantes respondieron las preguntas en la segunda sesión de estudio guiándose más por la estructura formal, lo que incidió en la baja correlación de las respuestas correctas sobre el aprendizaje.

Es también importante analizar que la cantidad absoluta de preguntas cuando se correlaciona con los resultados en la evaluación final tiene signo negativo (tabla 3). Este hecho pone de manifiesto que el factor crítico para el aprendizaje es la formulación preguntas que puedan ser respondidas correctamente por el estudiante. Se podría interpretar en términos, de que si hay errores, el sistema debería proveer condiciones para que se superen y haya mayor porcentaje de respuestas correctas. De acuerdo con investigaciones anteriores, es importante, en estos casos, sugerir estrategias de corrección, pues en esta circunstancia el estudiante está dispuesto a ponerlas en ejecución (Maldonado, et.al., 2001).

\section{Niveles de preguntas y aprendizaje de la habilidad cognitiva de hacer inferencias}

Los resultados apoyan más el aprendizaje específico de los niveles de inferencia, pero todavía no se pueden destacar niveles de generalización que serían menores que el nivel de aprendizaje específico. Las investigaciones analizadas por Holland, et.al., (1986) muestran diferencias entre el entrenamiento abstracto y formal en los procesos de inferencia y el entrenamiento vinculado a experiencias o significados específicos. Los usuarios aprenden mejor las formalidades cuando están asociadas con contornos prácticos o donde el significado tiene valor importante. Se requiere profundizar en este tema, por cuanto la formación de la capacidad cognitiva de hacer inferencias es muy importante en la formación de la capacidad para comprender y construir conocimientos científicos.

\section{REFERENCIAS BIBLIOGRÁFICAS}

ATKINSON, R. C., \& SHIFFRIN, R.M. (1968). Human Memory: A Proposed System and Its Control Processes. In K.W. Spence \& J.T. Spence (Eds.), The Psychology of Learning and Motivation, Vol. 2. New York: Academic Press.

AUSUBEL, D.P. (1968). Educational Psychology: A Cognitive View. New York: Holt, Rinehart, \& Winston.

BANGERT-DROWNS, R.L., KULIK, C.C., KULIK, J.A., MORGAN, M. (1991). The Instructional Effect of Feedback in Test-like Events. Review Educational Research, 61(2), 213-238.

BIEMANS, H.J.A. and SIMONS, R.J. (1999). Computer - assisted instructional strategies for promoting conceptual change. In New perspectives on conceptual change, edited by SCHNOTZ, W. And CARRETERO, M. Amsterdam: Pergamon Edit. 
FRANK, STEFAN L.; KOPPEN, MATHIEU; NOORDAM, LEO G.M.; AND VONK, WIETSKE (2003). Modeling Knowledge Based inferences in story comprehension. Cognitive Science, 27, 875-910.

FRANKLIN, S. \& GRAESSER, A. (1996). Is it an Agent, or just a Program?: A Taxonomy for Autonomous Agents. Proceedings of the Third International Workshop on Agent Theories, Architectures and Languages. Springer.

GAGNÉ, R. M. (1985). The Conditions of Learning and a Theory of Instruction. New York, N.Y.: Holt, Rinehart and Winston (fourth edition).

GAGNÉ, R. M., BRIGGS,L.J. \& WAGER, W.W. (1992). Principles of Instructional Design ( $4^{\text {th }}$ ed.). New York: Holt, Rinehart $\&$ Winston.

HOLLAND, JOHN H.; HOLYOAK, KEITH J.; NISBETT, RICHARD E.; AND THAGARD, PAUL R. (1986). Induction: processes of inference, learning and discovery. Cambridge, MA: The MIT Press.

KORIAT, ASHER AND SHITZER-REICHERT, RACHEL (2002). Metacognitive judgments and their accuracy. In Chambres, Patrick; Izaute, Marie; and Marescaux, Pierre-Jean (Editors): Metacognition: Process, function and use. Boston, MA: Kluwer Academic Publishers.

KUMAR, V.K. (1971). The Structure of Human Memory and Some Educational Implications. Review of Educational Research, 41(5), 379-417.

MALDONADO G. L. F.; ORTEGA, N., SANABRIA, R., L.B.; Y QUINTERO S. VICTOR J. (2003). Agente de software Q y el aprendizaje de modelos conceptuales. Tecné, Episteme y Didaxis, 14. 19-40.

MALDONADO G., L. F., ORTEGA, N., SANABRIA, R., L.B., MACIAS. M., D., GONZÁLEZ, D.P. (2001). Ambiente para el aprendizaje significativo: un ambiente para el diseño de hipertextos y evaluación del aprendizaje en ciencias sociales. Bogotá. Universidad Pedagógica Nacional y Colciencias.

MALDONADO G., L.F., FONSECA, R., O.H. IBAÑEZ, I., J. MACIAS, ORTEGA, N.,RUBIO, SANABRIA. R., L.B. (1999) Metacognición y razonamiento espacial en Juegos de Computador. -Bogotá: Instituto para la Investigación Educativa y el Desarrollo Pedagógico y Universidad Pedagógica Nacional.

MALDONADO G., L.F., ORTEGA, N., FONSECA, R., O.H., RUBIO, S., M. R., IBAÑEZ, I., J. y MACIAS. M., D. (2001). Razonamiento Espacial y Aprendizaje Significativo: profesores y alumnos frente a los juegos de descubrimiento basados en computador. Bogotá: Universidad Pedagógica Nacional y Colciencias. 
MALDONADO G., L.F., ORTEGA, N., SANABRIA, R., L.B. y MACIAS. M., D. (2003). Question generator software agents versus shells for building hypertext. Netherlands : Kluwer Academic Publishers.

MALDONADO, G. L.F., ORTEGA, N., FONSECA, O. H., SARMIENTO, L. C., GONZÁLEZ, D. N., MACIAS, M. D. e IBAÑEZ, I. J. (2001 c) La relación Simuladores y colaboración en el aprendizaje. Bogota: Instituto para la Investigación Educativa y el Desarrollo Pedagógico y Universidad Pedagógica Nacional.

MILLER, G.A.(1956). The Magical Number Seven, Plus or Minus Two: Some Limits on Our Capacity for Processing Information. The Psychology Review, 63(2), 81-97.

MINSKY, Marvin (1974). A framework for representing knowledge. In Haugeland, J. (1984), editor, Mind Design, pages 95-128, MIT Press, Cambridge MA. Reprinted in Brachman and Levesque (Eds).

MURDOCK, B. B. (1961): The retention of individual items. Journal of Experimental Psychology, 62, 618-625.

MURDOCK, B. B. (1965). A test of the limited capacity hypothesis. Journal of Experimental Psychology, 69, 237-240.

PFEIFER, R. (2001). Learning in Embodied Systems. Seminar in New Artificial Intelligence.

RAM, A. (1991). A theory of questions and question asking. The Journal of the Learning Sciences. 1 (3 \& 4), 273-318.

THIEDE, KEITH; ANDERSON, MARY C.; AND THERRIAULT, DAVID (2003). Accuracy of metacognitive monitoring affects learning of texts. Journal of Educational Psychology, 93, 1, 66-73.

WAGER, W. \& WAGER, S. (1985). Presenting Questions, Processing Responses and Providing Feedback in CAI. Journal of Instructional Development, 8(4), 2-8.

WEST, C.K., FARMER, J.A. \& WOLFF, P.M. (1991). Instructional Design: Implications from Cognitive Science. Englewood Cliffs, NJ: Prentice-Hall.

WOOLDRIDGE, M. (1997) “Agent-based software engineering” IEE Proc. On Software Engineering, 144 (1) 26-37. 